PROCEEDINGS OF THE

AMERICAN MATHEMATICAL SOCIETY

Volume 132, Number 4, Pages 1219-1222

S 0002-9939(03)07299-X

Article electronically published on November 7, 2003

\title{
ON GROUP OPERATIONS ON HOMOGENEOUS SPACES
}

\author{
YEVHEN ZELENYUK
}

(Communicated by Alan Dow)

\begin{abstract}
It is proved that every countably infinite homogeneous regular space admits a structure of any countably infinite group with continuous left shifts.
\end{abstract}

It is well known that not all infinite groups admit a non-discrete group topology (see, for example, [1, §9]). However, every infinite group admits a non-discrete zerodimensional topology with continuous left shifts [3], [4], and every countably infinite group admits a non-discrete zero-dimensional topology with continuous shifts and inversion [6]. It is well known also that not all homogeneous spaces admit a structure of a topological group and even a structure of a group with continuous left shifts (see, for example, $1, \S 10]$ ). The aim of this note is to prove that every countably infinite homogeneous regular space admits a structure of any countably infinite group with continuous left shifts.

We begin with the Boolean version of this result.

Theorem 1. Let $X$ be a countably infinite homogeneous regular space. Then there is a Boolean group operation + on $X$ with continuous shifts.

To prove Theorem 1 we need the following lemma.

Lemma. Let $X$ be a countably infinite homogeneous regular space and let $x, y \in$ $X, x \neq y$. Then there are a clopen neighborhood $U$ of $x$ and a homeomorphism $h: X \rightarrow X$ such that $h(x)=y, h(U)=X \backslash U$ and $h^{2}=i d_{X}$.

Proof. Enumerate $X$ as $\left\{x_{n}: n<\omega\right\}$. Since $X$ is homogeneous, there is a homeomorphism $g_{0}: X \rightarrow X$ with $g_{0}(x)=y$. Since $X$ is countable and regular, therefore zero-dimensional, one may choose a clopen neighborhood $U_{0}$ of $x$ with $U_{0} \cap g_{0}\left(U_{0}\right)=\emptyset$. Put $X_{0}=U_{0} \cup g_{0}\left(U_{0}\right)$ and define $h_{0}: X_{0} \rightarrow X_{0}$ by

$$
h_{0}(x)= \begin{cases}g_{0}(x) & \text { if } x \in U_{0} \\ g_{0}^{-1}(x) & \text { if } x \in g_{0}\left(U_{0}\right)\end{cases}
$$

If $X_{0}=X$, put $U=U_{0}$ and $h=h_{0}$. Otherwise, choose the first element $x_{n_{1}}$ in the sequence $\left\{x_{n}: n<\omega\right\}$ with $x_{n_{1}} \notin X_{0}$ and pick any $y_{n_{1}} \in X \backslash\left(X_{0} \cup\left\{x_{n_{1}}\right\}\right)$. Let $g_{1}: X \rightarrow X$ be a homeomorphism with $g_{1}\left(x_{n_{1}}\right)=y_{n_{1}}$ and let $U_{1}$ be a clopen

Received by the editors December 12, 2001 and, in revised form, May 23, 2002.

2000 Mathematics Subject Classification. Primary 22A30, 54H11; Secondary 20A05, 54A05.

Key words and phrases. Homogeneous space, Boolean group, left topological group, local isomorphism. 
neighborhood of $x_{n_{1}}$ with $U_{1} \cap g_{1}\left(U_{1}\right)=\emptyset$ and $U_{1} \cup g_{1}\left(U_{1}\right) \subseteq X \backslash X_{0}$. Put $X_{1}=$ $X_{0} \cup U_{1} \cup g_{1}\left(U_{1}\right)$ and extend $h_{0}$ to $h_{1}: X_{1} \rightarrow X_{1}$ by

$$
h_{1}(x)= \begin{cases}h_{0}(x) & \text { if } x \in X_{0}, \\ g_{1}(x) & \text { if } x \in U_{1}, \\ g_{1}^{-1}(x) & \text { if } x \in g_{1}\left(U_{1}\right) .\end{cases}
$$

If $X_{1}=X$, put $U=U_{0} \cup U_{1}$ and $h=h_{1}$. Otherwise, choose the first element $x_{n_{2}}$ in the sequence $\left\{x_{n}: n<\omega\right\}$ with $x_{n_{2}} \notin X_{1}$, pick any $y_{n_{2}} \in X \backslash\left(X_{1} \cup\left\{x_{n_{2}}\right\}\right)$, and so forth.

If $X_{n}=X$ for some $n$, put $U=\bigcup_{i \leq n} U_{i}$ and $h=h_{n}$. Otherwise, put $U=$ $\bigcup_{n<\omega} U_{n}$ and $h=\bigcup_{n<\omega} h_{n}$.

Proof of Theorem 1. We can assume that $X$ is not discrete. Pick any element $u \in X$ and enumerate the set $X \backslash\{u\}$ as $\left\{x_{n}: n<\omega\right\}$. By the Lemma, we can construct a decreasing sequence $\left\{U_{n}: n<\omega\right\}$ of clopen neighborhoods of $u$ with $U_{0}=X$ and a sequence of homeomorphisms $h_{n}: U_{n} \rightarrow U_{n}$ such that $h_{n}\left(U_{n+1}\right)=U_{n} \backslash U_{n+1}$ and $h_{n}^{2}=i d_{U_{n}}$. By induction on $n$, it is easy to verify that for each $n<\omega$ the subsets $h_{0}^{\varepsilon_{0}} \cdots h_{n}^{\varepsilon_{n}}\left(U_{n+1}\right)$, where $\varepsilon_{i} \in\{0,1\}$, form a partition of $X$ and every $x \in X$ can be uniquely written in the form $x=h_{0}^{\varepsilon_{0}} \cdots h_{n}^{\varepsilon_{n}}(y)$, where $y \in U_{n+1}$. We construct sequences $\left\{U_{n}: n<\omega\right\}$ and $\left\{h_{n}: n<\omega\right\}$ satisfying, in addition, the condition $x_{n} \in B_{n}=\left\{h_{0}^{\varepsilon_{0}} \cdots h_{n}^{\varepsilon_{n}}(u): \varepsilon_{i} \in\{0,1\}, i \leq n\right\}$. To see that this can be done, assume that $x_{n} \notin B_{n-1}$. Then $x_{n} \in h_{0}^{\varepsilon_{0}} \cdots h_{n-1}^{\varepsilon_{n-1}}\left(y_{n}\right)$ for some $\varepsilon_{i} \in\{0,1\}$ and $y_{n} \in U_{n}$. Choose $h_{n}$ with $h_{n}(u)=y_{n}$.

Now let $x, y \in X$. By the construction, there exists large enough $n$ such that $x$ and $y$ can be uniquely written in the form $x=h_{0}^{\varepsilon_{0}} \cdots h_{n}^{\varepsilon_{n}}(u), y=h_{0}^{\delta_{0}} \cdots h_{n}^{\delta_{n}}(u)$. Put $x+y=h_{0}^{\varepsilon_{0}+\delta_{0}} \cdots h_{n}^{\varepsilon_{n}+\delta_{n}}(u)$. It is clear that the operation is well defined and that $(X,+)$ is a Boolean group with zero $u$. We note that for every $z \in U_{n+1}$, $x+z=h_{0}^{\varepsilon_{0}} \cdots h_{n}^{\varepsilon_{n}}(z)$. To check that the left shifts in $(X,+)$ are continuous, let $U$ be a neighborhood of $x+y$. Choose a neighborhood $V$ of $u$ such that $V \subseteq U_{n+1}$ and $h_{0}^{\varepsilon_{0}+\delta_{0}} \cdots h_{n}^{\varepsilon_{n}+\delta_{n}}(V) \subseteq U$. Then $W=h_{0}^{\delta_{0}} \cdots h_{n}^{\delta_{n}}(V)$ is a neighborhood of $y$ and for every $z \in V, x+h_{0}^{\delta_{0}} \cdots h_{n}^{\delta_{n}}(z)=x+(y+z)=(x+y)+z=h_{0}^{\varepsilon_{0}+\delta_{0}} \cdots h_{n}^{\varepsilon_{n}+\delta_{n}}(z) \in U$; so $x+W \subseteq U$.

Next we need the Local Isomorphism Theorem. It is close to [5. Theorem 2] (see also [2, Lemma 7.4]).

A space with a group operation is called a left topological group if all left shifts are continuous. A space $X$ with a partial binary operation - and a distinguished element 1 is called a local left topological group, if for each element $x \in X$ there is an open neighborhood $U_{x}$ of 1 such that

(1) for any $y \in U_{x}, x \cdot y$ is defined, $x \cdot 1=x, x \cdot U_{x}$ is an open neighborhood of $x$, and a mapping $U_{x} \ni y \mapsto x \cdot y \in x \cdot U_{x}$ is a homeomorphism;

(2) $(x \cdot y) \cdot z=x \cdot(y \cdot z)$ if $y \in U_{x}, z \in U_{x \cdot y} \cap U_{y}, y \cdot z \in U_{x}$.

For a local left topological group, from this point on, when we write $x \cdot y$ we mean $y \in U_{x}$ and when we write $x \cdot U$, where $U$ is a neighborhood of 1 , we mean $U \subseteq U_{x}$. A basic example of a local left topological group is an open neighborhood of the identity of a left topological group.

Let $X$ and $Y$ be local left topological groups. A map $f: X \rightarrow Y$ is called a local homomorphism if $f\left(1_{X}\right)=1_{Y}$ and for every $x \in X$ there exists a neighborhood $U_{x}$ 
of $1_{X}$ such that $f(x z)=f(x) f(z)$ for all $z \in U_{x}$. A bijective local homomorphism $f$ is called a local isomorphism if $f^{-1}$ is also a local homomorphism. We observe that every open bijective local homomorphism is a local isomorphism.

Theorem 2. All countably infinite non-discrete regular left topological groups are local isomorphic.

Proof. Let $X$ be the countably infinite Boolean group $\bigoplus_{\omega} \mathbb{Z}_{2}$ endowed with the direct sum topology, and let $Y$ be an arbitrary countably infinite non-discrete regular left topological group. We shall define a local isomorphism $f: X \rightarrow Y$.

Let $F$ be the semigroup of words on the letters 0 and 1 with empty word $\emptyset$, and let $F^{\prime}$ be the subsemigroup of $F$ of words including $\emptyset$, in which the last letter is 1. We define a bijection $X \ni x \mapsto w(x) \in F^{\prime}$ as follows. If $0 \neq x=\left(\varepsilon_{n}\right)_{n<\omega}$ and $m=\max \left\{n<\omega: \varepsilon_{n}=1\right\}$, we put $w(x)=\varepsilon_{0} \cdots \varepsilon_{m}$. If $x=0$, we put $w(x)=\emptyset$.

For every $w \in F,|w|$ will denote the length of $w$. For every $n<\omega$, put $W_{n}=$ $\{w \in F:|w|=n\}$. Each nonempty $w \in F$ has a unique representation in the form $w=w_{1} w_{2} \cdots w_{k}$, where $w_{l}=0^{i_{l}} 1^{j_{l}}, 1 \leq l \leq k, i_{1}, j_{k} \in \omega, j_{1}, i_{2}, j_{2}, \ldots, i_{k} \in \mathbb{N}$ (if $k=1$, the requirement is $i_{1}, j_{1} \in \omega$ and $i_{1}+j_{1} \in \mathbb{N}$ ). This representation will be called canonical. Words of the form $0^{i} 1^{j}$, where $i, j \in \omega$ and $i+j \in \mathbb{N}$, will be called basic. If a word $w$ is basic or $w=\emptyset$, we put $w^{\prime}=\emptyset$ and $w^{*}=w$. Otherwise, if $w=w_{1} w_{2} \cdots w_{k}$ is the canonical representation, we put $w^{\prime}=w_{1} w_{2} \cdots w_{k-1}$ and $w^{*}=0^{\left|w_{1}\right|+\cdots+\left|w_{k-1}\right|} w_{k}$.

Enumerate $Y \backslash\left\{1_{Y}\right\}$ as $\left\{y_{n}: 0<n<\omega\right\}$. We shall construct a clopen $Y(w) \subseteq Y$ and $y(w) \in Y(w)$ for every $w \in F$ such that $Y(\emptyset)=Y, y(\emptyset)=1_{Y}$ and the following conditions hold for all $n \in \mathbb{N}$ :

(1) $Y(w 0) \cup Y(w 1)=Y(w)$ and $Y(w 0) \cap Y(w 1)=\emptyset$ for all $w \in W_{n-1}$;

(2) $y(w 0)=y(w)$ for all $w \in W_{n-1}$;

(3) $Y(w)=y\left(w^{\prime}\right) Y\left(w^{*}\right)$ and $y(w)=y\left(w^{\prime}\right) y\left(w^{*}\right)$ for all $w \in W_{n}$;

(4) $y_{n} \in\left\{y(w): w \in W_{n}\right\}$.

We take as $Y(0)$ a clopen neighborhood of $1_{Y}$ such that $y_{1} \notin Y(0)$. Put $Y(1)=$ $Y \backslash Y(0), y(0)=1_{Y}$ and $y(1)=y_{1}$.

Suppose that $Y(w)$ and $y(w)$ have already been constructed for all $w \in W_{n}$ such that conditions (1)-(4) hold.

It is obvious that the subsets $Y(w)$, where $w \in W_{n}$, form a partition of $Y$. So, one of them, say $Y(u)$, contains $y_{n+1}$. For some $z_{n+1} \in Y\left(u^{*}\right), y_{n+1}=y\left(u^{\prime}\right) z_{n+1}$. If $z_{n+1}=y\left(u^{*}\right)$, we take as $Y\left(0^{n+1}\right)$ any clopen neighborhood of $1_{Y}$ such that $Y(w) \backslash y(w) Y\left(0^{n+1}\right) \neq \emptyset$ for all basic $w \in W_{n}$. Then for every basic $w \in W_{n}$, put $y(w 0)=y(w), Y(w 0)=y(w) Y\left(0^{n+1}\right)$ and $Y(w 1)=Y(w) \backslash Y(w 0)$, and take as $y(w 1)$ any element of $Y(w 1)$. If $z_{n+1} \neq y\left(u^{*}\right)$, we take as $Y\left(0^{n+1}\right)$ in addition such that $z_{n+1} \notin y\left(u^{*}\right) Y\left(0^{n+1}\right)$ and put $y\left(u^{*} 1\right)=z_{n+1}$. For all non-basic $v \in W_{n+1}$, we define $Y(v)$ and $y(v)$ by condition (3). Then $y(v)=y\left(v^{\prime}\right) y\left(v^{*}\right) \in y\left(v^{\prime}\right) Y\left(v^{*}\right)=Y(v)$ and $y_{n+1}=y\left(u^{\prime}\right) y\left(u^{*} 1\right)=y\left((u 1)^{\prime}\right) y\left((u 1)^{*}\right)=y(u 1)$. To check conditions (2) and (1), let $w \in W_{n}$. Then

$$
\begin{gathered}
y(w 0)=y\left((w 0)^{\prime}\right) y\left((w 0)^{*}\right)=y(w) y\left(0^{n+1}\right)=y(w), \\
Y(w 0)=y(w) Y\left(0^{n+1}\right)=y\left(w^{\prime}\right) y\left(w^{*}\right) Y\left(0^{n+1}\right)=y\left(w^{\prime}\right) Y\left(w^{*} 0\right), \\
Y(w 1)=y\left((w 1)^{\prime}\right) Y\left((w 1)^{*}\right)=y\left(w^{\prime}\right) Y\left(w^{*} 1\right)
\end{gathered}
$$


and so

$$
\begin{gathered}
Y(w 0) \cup Y(w 1)=y\left(w^{\prime}\right)\left[Y\left(w^{*} 0\right) \cup Y\left(w^{*} 1\right)\right]=y\left(w^{\prime}\right) Y\left(w^{*}\right)=Y(w), \\
Y(w 0) \cap Y(w 1)=\emptyset .
\end{gathered}
$$

After this construction we have obtained the mapping $F^{\prime} \ni w \mapsto y(w) \in Y$. It follows from (4), (2) and (1) that it is a bijection. Define the bijection $f: X \rightarrow Y$ by $f(x)=y(w(x))$. To verify that $f$ is a local homomorphism, let $x \in X, w(x)=u$. Take any $z \in U_{|u|+1}$, where $U_{n}=\left\{\left(\varepsilon_{i}\right)_{i<\omega} \in X: \varepsilon_{i}=0\right.$ for all $\left.i<n\right\}$. Then $w(z)=0^{|u|+1} v$ and $w(x+z)=u 0 v$. It is easy to show by induction on the length of the canonical representations using (3) that $y(u 0 v)=y(u) y\left(0^{|u|+1} v\right)$. Therefore, $f(x+z)=y(u 0 v)=y(u) y\left(0^{|u|+1} v\right)=f(x) f(z)$. To see that $f$ is a local isomorphism, we note that $f\left(U_{n+1}\right)=Y\left(0^{n}\right)$; so $f$ is open.

Now we can prove our main result.

Theorem 3. Let $X$ be a countably infinite homogeneous regular space, and let $G$ be a countably infinite group. Then there is a group operation $*$ on $X$ with continuous left shifts such that $(X, *)$ is isomorphic to $G$.

Proof. We may suppose that $X$ is not discrete. By Theorem 1, there is a Boolean group operation + on $X$ with continuous shifts. We endow $G$ with any non-discrete regular topology with continuous left shifts. By Theorem 2, there is a local isomorphism $f:(X,+) \rightarrow G$ (it even suffices that $f$ be a bijective local homomorphism). For any $x, y \in X$, we define $x * y=f^{-1}(f(x) f(y))$. Obviously, $(X, *)$ is a group isomorphic to $G$. Next, given any $x \in X$, we can choose a neighborhood $U$ of the identity such that $f(x+z)=f(x) f(z)$ for all $z \in U$, and then $x * z=f^{-1}(f(x) f(z))=f^{-1}(f(x+z))=x+z$. It follows from this that the left shifts of $(X, *)$ are continuous and open at the identity. Consequently, the left shifts of $(X, *)$ are continuous.

\section{REFERENCES}

1. W. Comfort, Topological groups, in Handbook of Set-theoretic Topology, edited by K. Kunen and J. E. Vaughan, North-Holland, Amsterdam, 1984, 1143-1263. MR 86g:22001

2. N. Hindman and D. Strauss, Algebra in the Stone-Čech compactification, De Gruyter, Berlin, 1998. MR 99j:54001

3. T. Papazyan, Extremal Topologies on a Semigroup, Topology and its Applications 39 (1991), 229-243. MR 92f:22003

4. I. Protasov, Filters and topologies on semigroups, (in Russian) Mat. Studii 3 (1994), 15-28. MR 2000k:22005

5. Y. Zelenyuk, Finite Groups in $\beta \mathbb{N}$ are Trivial, Semigroup Forum 55 (1997), 131-132. MR 99b:22004

6. Y. Zelenyuk, On topologies on groups with continuous shifts and inversion, (in Ukrainian) Visnyk Kyiv Univ., Ser. Fiz.-Mat. No. 2 (2000), 252-256.

Faculty of Cybernetics, Kyiv Taras Shevchenko University, vul. Glushkova 2, korp. 6, 03680, KYIV, UKRAINE

E-mail address: grishko@i.com.ua

URL: http://www.i.com.ua/ grishko/zelenyuk.html 